Revista Energia na Agricultura

ISSN 1808-8759

\title{
AVALIAÇÃO DE UM SENSOR DE CAPACITÂNCIA ELÉTRICA E SUA CORRELAÇÃO COM ATRIBUTOS DO SOLO ${ }^{1}$ \\ VICTOR GEORGE CELINSKI² \& CÉLIA REGINA LOPES ZIMBACK ${ }^{3}$
}

RESUMO: As medidas elétricas do solo têm sido utilizadas como variáveis que se correlacionam com atributos do solo. O presente trabalho teve como objetivos: desenvolver um sensor de capacitância elétrica de baixo custo; avaliar seu desempenho em campo; e, verificar a correlação entre a leitura de capacitância elétrica com propriedades físicas (areia, silte e argila), propriedades químicas do solo (pH, M.O., P resina, $\mathrm{H}+\mathrm{Al}, \mathrm{K}, \mathrm{Ca}, \mathrm{Mg}, \mathrm{SB}, \mathrm{CTC}$ e V\%) e o teor de água. A coleta dos dados foi realizada na Fazenda Capão da Onça, pertencente à Universidade Estadual de Ponta Grossa, em uma área com aproximadamente 13 hectares, totalizando 81 amostras. Em cada ponto amostral foi medida a capacitância elétrica do solo. Após as leituras e a retirada do sensor, foram coletadas amostras do solo, analisadas no laboratório da Faculdade de Ciências Agronômicas da Universidade Estadual Paulista. O instrumento de medida utilizado para a coleta dos dados de capacitância elétrica do solo foi um multímetro digital. Os dados foram submetidos às análises de correlação e regressão. O sistema desenvolvido apresentou um baixo custo e foi capaz de medir as variações da capacitância elétrica do solo. As medidas de capacitância elétrica obtidas correlacionaram-se satisfatoriamente com os teores de argila e areia, e fracamente com o teor de umidade. Isto demonstrou a possibilidade de uso do sensor para verificar a textura do solo em áreas não homogêneas. As medidas de capacitância elétrica do solo também se correlacionaram significativamente com os atributos do solo: cálcio, magnésio, pH, SB e CTC. Estes resultados demonstraram a possibilidade de o sensor ser utilizado no controle da fertilidade do solo.

Palavras-chave: Agricultura de precisão, sensor de medidas elétricas.

\footnotetext{
${ }^{1}$ Parte da tese de doutorado do $1^{\circ}$. autor intitulada: Desenvolvimento de sensor elétrico de contato e correlações com atributos do solo visando à agricultura de precisão.

${ }^{2}$ Aluno do curso de PG Energia na Agricultura - FCA/UNESP - Botucatu/SP - Brasil. Docente na Universidade Estadual de Ponta Grossa. vgcelinski@gmail.com

${ }^{3}$ Orientadora e docente do Departamento de Recursos Naturais - FCA/UNESP - Botucatu/SP.
} 


\section{EVALUATION OF A ELECTRICAL CAPACITANCE SENSOR AND CORRELATION WITH SOIL ATTRIBUTES}

SUMMARY: The electrical measures of the soil have been used as variables that correlate with its characteristics. This study aimed at developing an electrical capacitance sensor of low cost, to evaluate its performance on the field and verify the correlation between the measurements of electrical capacitance with physical properties (sand, silt and clay) and chemical properties of soil ( $\mathrm{pH}, \mathrm{MO}, \mathrm{P}$ resin, $\mathrm{H}+\mathrm{Al}, \mathrm{K}$, $\mathrm{Ca}, \mathrm{Mg}, \mathrm{SB}, \mathrm{CTC}$ and $\mathrm{V} \%)$ and the moisture content. The data sampling was performed at the farm named "Capão da Onça" which belongs to the State University of Ponta Grossa. The samples collection was conducted in an area of approximately 13 hectares, totalizing 81 samples. In each sampling the electrical capacitance of the soil was measured. After the sensor withdrawal, soil samples were collected and sent to be analysed in the laboratory of the College of Agronomics Science of the Paulista State University. The measuring instrument used to collect data on electric capacitance of the soil a digital multimeter was used. The data were submitted to the analysis of correlation and regression. The developed system presented a low cost and it was capable to measuring variation of the electrical capacitance of the soil. The obtained measures satisfactorily correlated with the levels of clay and sand, and weakly with the moisture content. This had demonstrated the possibility to use a sensor to verify the soil texture in not homogeneous areas. The measures of the electrical capacitance of the soil obtained by the sensor had significantly correlated with the soil attributes: calcium, magnesium, $p H, S B$ and CTC. These results had demonstrated the possibility to use a sensor for soil fertility control.

Keywords: Precision farming, sensor of electric measures.

\section{INTRODUÇÃO}

Os elevados níveis de produtividade agrícola estão associados ao uso intenso de insumos (PINCELLI, 2004). Diante disto, há a necessidade de se encontrar novos conceitos que permitam a sua redução e consequientemente os custos de produção e o impacto ambiental, além de incrementar a produção. A busca a esse novo meio de produção agrícola tem levado ao conceito de agricultura de precisão, que pode ser entendida como um conjunto de técnicas que permitem o manejo diferenciado das áreas agrícolas, voltadas às suas reais necessidades, pelo uso das técnicas agronômicas e a aplicação localizada de insu- 
mos. As técnicas de agricultura de precisão surgiram com a finalidade de otimizar a produção agrícola, não só reduzindo os custos como aumentando a produtividade.

Desde os primórdios do século XX, alguns pesquisadores procuravam desenvolver uma metodologia para que os produtores pudessem amostrar, testar e mapear os solos, de forma simples e prática e que resultasse numa economia dos insumos aplicados (GUERRA, 2006).

Para Grego et al. (2006), a investigação de possíveis causas da variabilidade espacial encontrada nos resultados de produtividade das culturas tem sido atribuída aos fatores de solo, como as propriedades físicas e químicas. Entretanto, estas são obtidas por amostragens que, na maioria, exigem alta demanda de mão-de-obra, de tempo e de custo. As medidas elétricas do solo têm chamado a atenção, principalmente, por ser obtida através de métodos eficientes e rápidos como pelos sensores de contato direto com o solo.

Rabello et al. (2005) relataram que os métodos que utilizam as propriedades dielétricas são bastante utilizados atualmente, consistindo em um grande número de sistemas comerciais com diversas características específicas de design, resolução, precisão e preço. Dentre as técnicas dielétricas, são citadas as que utilizam o princípio da Reflectometria no Domínio do Tempo (Team Domain Reflectometry - TDR) e a capacitância elétrica.

Para Placido (2005), o sensor genérico pode ser um instrumento, onde o sinal de entrada pode ser uma quantidade, uma propriedade ou um estado, que o sensor converte em uma quantidade elétrica (carga, tensão ou corrente elétrica) e que pode ser amplificada e modificada por circuitos eletrônicos de aquisição e controle.

Adamchuk et al. (2004) relataram que embora exista uma grande variedade de sensores do solo, a maioria deles envolve um dos seguintes métodos de medição: os sensores elétricos e eletromagnéticos, que medem a resistividade, a condutividade, a capacitância ou a indutância elétrica, sendo afetados pela composição do solo. A maioria dos sensores fornece um sinal de saída, que é afetado por mais de uma característica agronômica do solo. Sensores elétricos e eletromagnéticos são instrumentos de medida baseados em circuitos elétricos e usados para determinar a habilidade de determinados meios em conduzir ou acumular a carga elétrica. Se o solo for usado como tal meio, suas características físicas e químicas podem afetar o comportamento do circuito e, assim, os parâmetros elétricos medidos.

Desta forma, faz-se necessário o desenvolvimento de sensores que realizem determinações com maior rapidez e que possuam boa correlação com o método padrão. O presente estudo teve como objetivos projetar um sensor de capacitância elétrica, avaliar seu desempenho em campo e verificar a correlação entre as leituras de capacitância elétrica com alguns atributos físicos (areia, silte e argila), atributos químicos do solo (pH, M.O., Presina, H+Al, K, Ca, Mg, SB, CTC e V\%), condutividade elétrica em laboratório e o teor de água. 


\section{MATERIAL E MÉTODOS}

\section{1 Área de estudo}

A coleta dos dados da pesquisa foi realizada em uma gleba da Fazenda Capão da Onça, pertencente à Universidade Estadual de Ponta Grossa, localizada na região dos Campos Gerais, no município de Ponta Grossa, no estado do Paraná. Em um Cambissolo Háplico distrófico de textura média. O clima da região, segundo Köeppen, é do tipo Cfb, sempre úmido, quente temperado, sem estação seca definida e com geadas frequientes no inverno e temperaturas médias de $22^{\circ} \mathrm{C}$ no verão. A precipitação anual é em torno de $1.422 \mathrm{~mm}$ e a umidade relativa do ar de $75 \%$, em média.

\subsection{Montagem do sensor de capacitância elétrica}

O sensor de capacitância elétrica desenvolvido no estudo tem o princípio de funcionamento semelhante ao método dos dois eletrodos, conforme foi exposto por Pincelli (2004). O processo de montagem do sensor seguiu seu desenvolvimento pela escolha de alternativas práticas e de baixo custo. Procurou-se, então, por um material que fosse menos susceptível à deformações. Foi adotada a opção de utilizar tubos de cobre e de PVC, com 2,2 cm de diâmetro, utilizado em sistemas de água quente residenciais.

Foram realizados vários testes com diversas medidas dos eletrodos e do isolador. Verificou-se que o melhor resultado foi dimensionar o sensor com $5 \mathrm{~cm}$ de comprimento para cada eletrodo e também o isolador, formando uma haste de $15 \mathrm{~cm}$ de comprimento e $2,2 \mathrm{~cm}$ de diâmetro para aplicação vertical no solo, com uma das extremidades com forma pontiaguda para facilitar a penetração no solo, confeccionada com material isolante de uma resina acrílica, como pode ser observado na Figura 1.

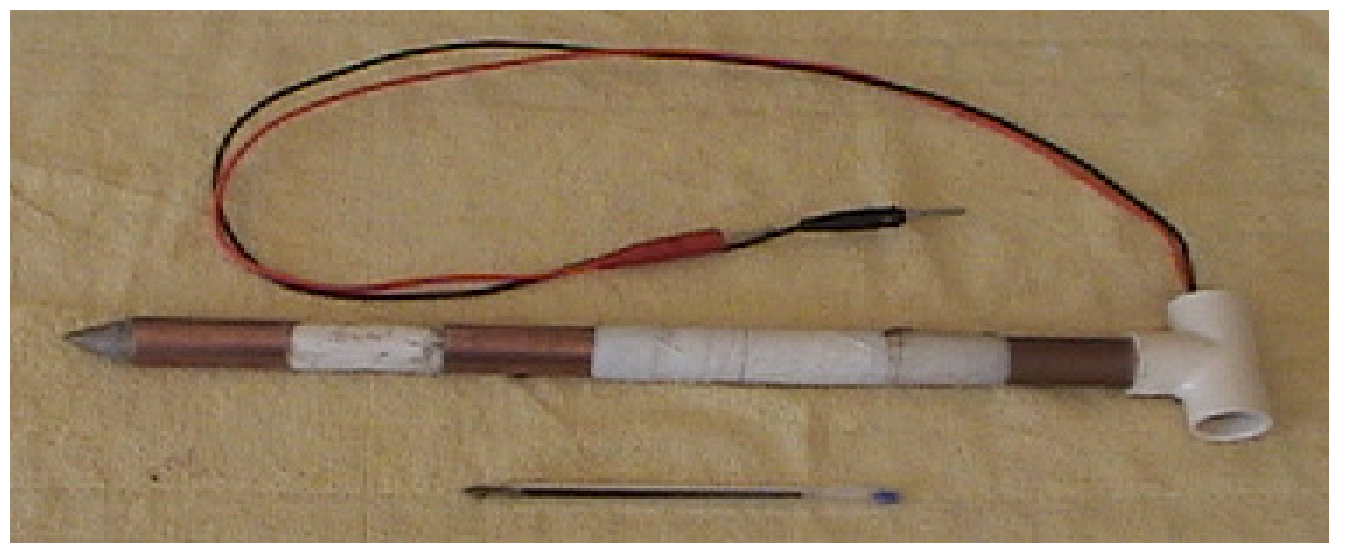

Figura 1 - Sensor de capacitância elétrica. 


\subsection{Medidas de capacitância elétricas do solo}

Para realizar as medidas de capacitância elétrica do solo com o sensor em estudo, foi utilizado um multímetro digital com capacímetro. Este método é semelhante ao utilizado por Rabello et al. (2005), onde as medidas do sensor são obtidas por meio de um multímetro. As leituras dos valores de capacitância elétrica, e também a coleta das amostras do solo, foram realizadas no período de novembro à dezembro de 2006, imediatamente após a colheita da cultura do trigo.

O sensor foi introduzido verticalmente no solo até $20 \mathrm{~cm}$ de profundidade. Essa medida foi escolhida porque é nela que se situa a faixa onde a maior parte das raízes se concentra. Para proteger e facilitar a colocação do sensor no solo foi utilizado um instrumento pré-furador, confeccionado com as mesmas dimensões e o mesmo tubo de cobre usado na montagem do sensor. As medidas de capacitância elétrica foram realizadas para cada ponto amostral, antecedendo imediatamente a coleta das amostras do solo.

\subsection{Amostragem do solo}

A coleta das amostras do solo foi realizada em uma área comercial de plantio direto com aproximadamente 13 hectares, totalizando 81 amostras, resultando em aproximadamente 6,2 leituras por hectare. Foi estabelecido um plano espacial determinado, mantendo uma regularidade na coleta de amostras por toda a área. As distâncias entre as amostras não foram iguais, mas a posição relativa de cada amostra foi georreferenciada com aparelho GPS portátil. As amostras foram coletadas manualmente, com trado de 10 $\mathrm{cm}$ de diâmetro, na profundidade de 5 a $20 \mathrm{~cm}$, obedecendo a uma seqüência sistemática em cada ponto de amostragem. Na profundidade de 0 a $5 \mathrm{~cm}$ não foram coletadas amostras, considerando que nesta profundidade o contato com o sensor seria prejudicado por apresentar-se deficiente. Foi observado que a colocação do sensor no solo aumenta em alguns milímetros o diâmetro de contato da superfície do solo com o sensor.

Logo após a retirada do sensor do solo, em cada ponto amostral foi coletada uma amostra de 1,2 $\mathrm{kg}$ de solo, a qual foi colocada num balde. Em seguida, para cada amostra, uma sub-amostra foi retirada do balde, na quantidade de $0,2 \mathrm{~kg}$ de solo, acondicionada em embalagem própria de alumínio, devidamente lacrada. Outra quantidade de $0,2 \mathrm{~kg}$ foi retirada e colocada em saco plástico específico, identificado pelo número do ponto amostral respectivo. Este conjunto de amostras foi levado ao Laboratório de Solos da Universidade Estadual de Ponta Grossa para realizar as análises dos teores de água e a condutividade elétrica, conforme metodologia preconizada para tais atributos (EMBRAPA, 1997). 
O restante da amostra foi colocado em saco plástico específico, identificado pelo número do ponto amostral, e levado para análise química (macro nutrientes) e análise física (areia, silte e argila) nos Laboratórios da Faculdade de Ciências Agronômicas, da Universidade Estadual Paulista, Campus de Botucatu.

\subsection{Análise dos dados}

Para analisar a correlação das leituras de capacitância elétrica medidas pelo sensor em relação aos atributos físicos-químicos, condutividade elétrica em laboratório e o teor de água do solo, foram utilizados os resultados das amostras do solo analisados nos laboratórios referenciados anteriormente.

As amostras foram analisadas para determinar as características químicas de: potencial de hidrogênio $(\mathrm{pH})$, acidez potencial (H+Al), matéria orgânica (M.O.), fósforo extraído da resina (Presina), cálcio $(\mathrm{Ca})$, magnésio $(\mathrm{Mg})$, potássio $(\mathrm{K})$, e os seguintes parâmetros: soma de bases $(\mathrm{SB})$, capacidade de troca de cátions (CTC), condutividade elétrica (Ce), teor de água e saturação por bases (V\%). Da mesma forma, para determinar as características físicas de: teor de areia, teor de silte e teor de argila. As metodologias de determinação dos teores desses atributos do solo foram as mesmas utilizadas por (RAIJ et al., 2001).

Com a finalidade de estimar os valores dos atributos do solo por meio dos valores das medidas de capacitância elétrica levantados em campo, foi realizada a análise de regressão com o gráfico da linha de tendência, apresentando a expressão matemática da relação e o coeficiente de determinação das variáveis. $\mathrm{O}$ coeficiente de determinação $\left(\mathrm{r}^{2}\right)$ é o índice que resulta a percentagem de variação de uma variável que é explicada estatisticamente pela variação na outra variável. Para o cálculo destes parâmetros foi utilizado o programa Microsoft Excel 2003. Segundo Vanni (1998), o coeficiente de determinação pode ser interpretado pelo sentido relativo quando assumir valores entre 0 e 1 , revelando um percentual de ajustamento, onde um ajustamento entre 0,65 e 0,75 pode ser considerado regular, entre 0,75 e 0,85 pode ser considerado bom e acima de 0,85 deve ser considerado ótimo. Abaixo de 0,6 demonstra que a variável independente "x" não explica com segurança a variação de "y". Para análise da distribuição dos pontos nos gráficos foi utilizada a metodologia conforme Doria Filho (1999), que classificou os coeficientes de correlação linear entre variáveis como perfeito, quando o índice de correlação for $(=1)$, forte $(>0,75)$, médio $(>0,5)$, fraco $(<0,5)$ e inexistente $(=0)$. 


\section{RESULTADOS E DISCUSSÃO}

\subsection{Sensor de capacitância elétrica}

O sensor do presente estudo resultou num sistema mecânico e eletrônico para a realização de sondagens dos atributos do solo no campo, de forma simples, rápida e de baixo custo. Sua principal vantagem é a de poder ser inserido no solo de forma vertical, como uma lança, e retirado com facilidade, proporcionando uma deformação mínima do solo (pequeno orifício de cerca de $2,2 \mathrm{~cm}$ de diâmetro). Como desvantagem, observa-se que a introdução do sensor em solos compactados necessita de um instrumento préfurador do solo.

\subsection{Análise dos coeficientes de determinação e dispersão dos dados}

Os resultados da análise de regressão com o gráfico da linha de tendência, com a expressão matemática da relação e o coeficiente de determinação das variáveis podem ser visualizados nas Figuras 2 a 9.
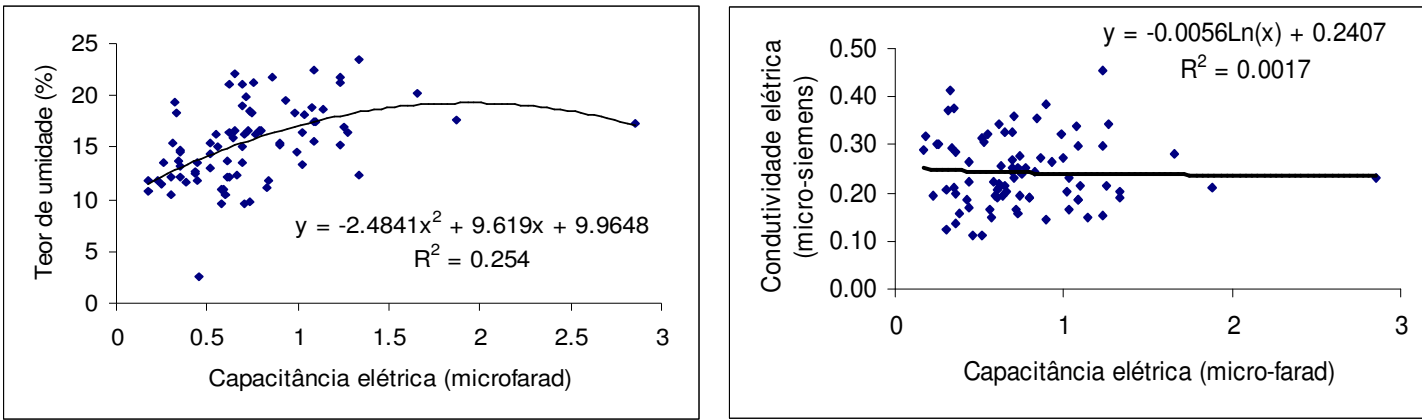

Figura 2 - Gráficos de dispersão e linha de tendência entre os valores de capacitância elétrica com o teorde umidade (a) e com a condutividade elétrica (b).

Pode-se observar na Figura 2 que os coeficientes de determinação de ambos os gráficos, conforme Vanni (1998), não explicam com segurança as variações do teor de umidade e condutividade elétrica em função da capacitância elétrica, e a distribuição dos pontos no gráfico da Figura 2 (a) se apresenta dispersa, indicando uma média correlação entre as variáveis. No gráfico da Figura 2 (b), a distribuição dos pontos se apresenta muito dispersa, indicando uma fraca correlação entre as variáveis, segundo a classificação adotada por (DORIA FILHO, 1999). Analisando a Figura 2 (a), nota-se que quanto maior o teor de umi- 
dade maior o índice de capacitância elétrica do solo, ou seja, estes apresentaram uma relação semelhante ao estudo realizado por Green et al. (2005) que também realizaram uma experiência para melhorar o conhecimento sobre o comportamento e características de um sensor capacitivo e verificaram que o conteúdo de água do solo pode ser estimado diretamente a partir da permissividade dielétrica do solo. Com o objetivo de caracterizar o comportamento de sensores capacitivos para monitorar a umidade do solo, Rende e Biage (2002) verificaram que o comportamento dos sensores capacitivos tinha características especificas para cada tipo de solo, sendo assim necessário desenvolver curvas de calibração para cada tipo de solo.

Adotou-se para as demais análises dos coeficientes de determinação e dispersão dos dados a mesma metodologia utilizada anteriormente, ou seja, coeficiente de determinação conforme Vanni (1998) e índice de correlação segundo (DORIA FILHO, 1999).
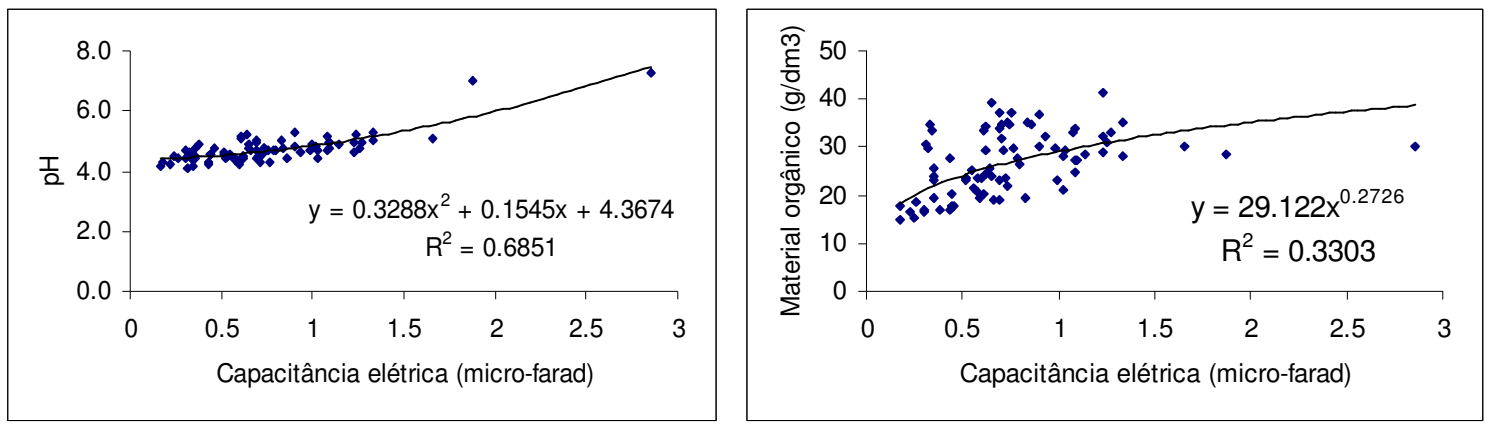

Figura 3 - Gráficos de dispersão e linha de tendência entre os valores de capacitância elétrica com o pH (a) e com matéria orgânica (b).

Verifica-se na Figura 3 (a) que o coeficiente de determinação em relação à capacitância elétrica explica com regular segurança as variações do índice de $\mathrm{pH}$ e a distribuição dos pontos no gráfico se apresenta quase sem dispersão, indicando uma forte correlação entre as variáveis. Na Figura 3 (b) o coeficiente de determinação do gráfico não explica com segurança a variação do teor de material orgânico em função da capacitância elétrica e a distribuição dos pontos no gráfico se apresenta dispersa, indicando uma média correlação entre as variáveis. 

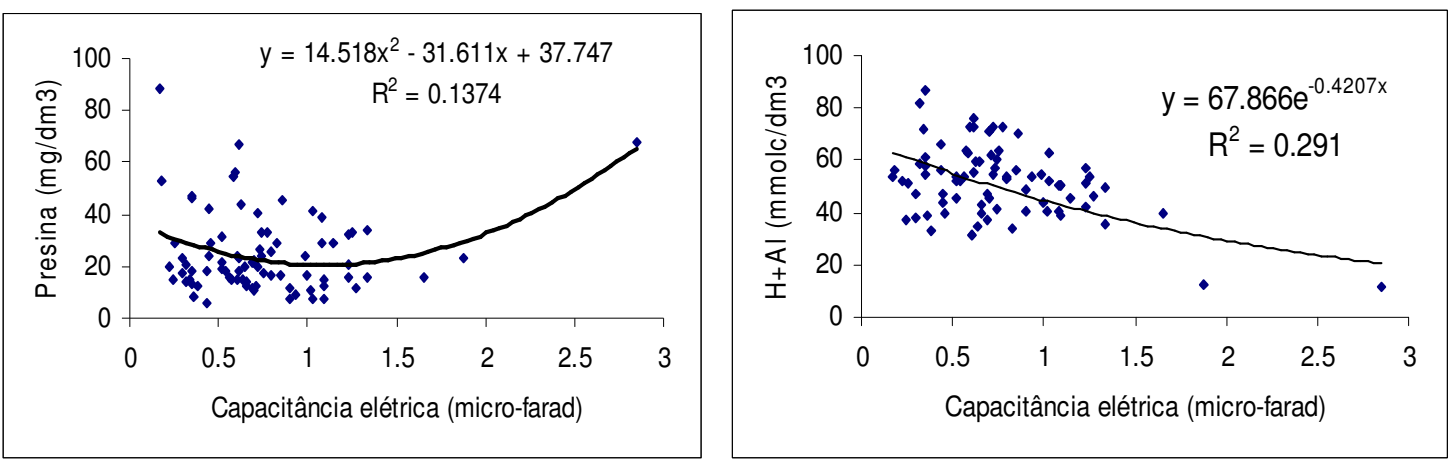

Figura 4 - Gráficos de dispersão e linha de tendência entre os valores de capacitância elétrica com Presina (a) e com $\mathrm{H}+\mathrm{AL}$ (b).

Pode-se observar na Figura 4 que os coeficientes de determinação de ambos os gráficos não explicam com segurança as variações da capacitância elétrica em função do teor de Presina e H+Al e a distribuição dos pontos no gráfico da Figura 4 (a) se apresenta muito dispersa, indicando uma fraca correlação entre as variáveis. Observa-se, na Figura 4 (b), que a distribuição dos pontos no gráfico se apresenta dispersa, indicando uma média correlação entre as variáveis.
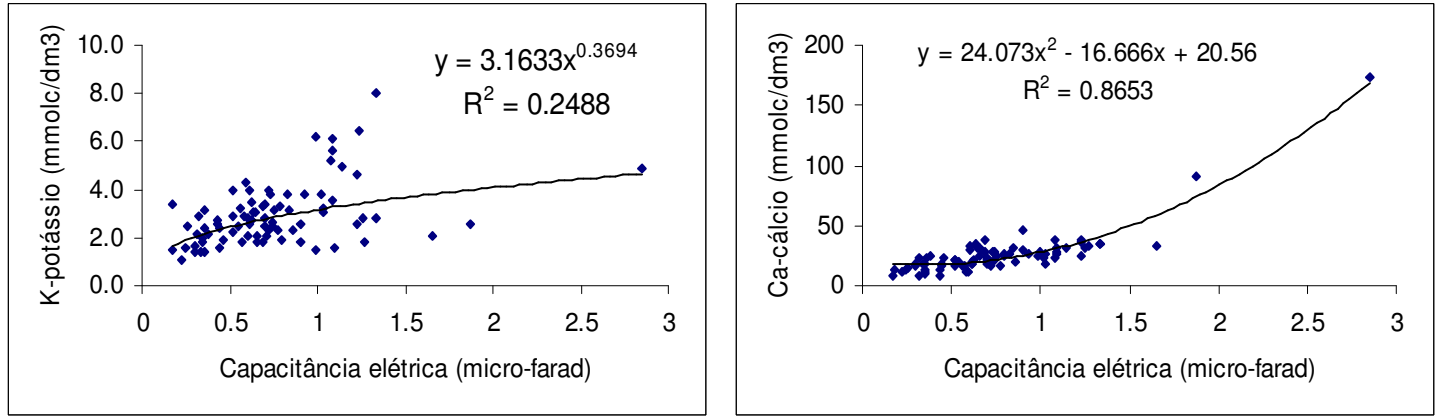

Figura 5 - Gráficos de dispersão e linha de tendência entre os valores de capacitância elétrica com potássio (a) e com cálcio (b).

Pode-se verificar na Figura 5 (a) que o coeficiente de determinação do gráfico não explica com segurança as variações do teor de potássio em função da capacitância elétrica e a distribuição dos pontos no gráfico se apresenta muito dispersa, indicando uma fraca correlação entre as variáveis. Na Figura 5 (b), o coeficiente de determinação do gráfico explica com ótima segurança as variações do índice de cálcio e a 
distribuição dos pontos no gráfico se apresenta quase sem dispersão, indicando uma forte correlação entre as variáveis.
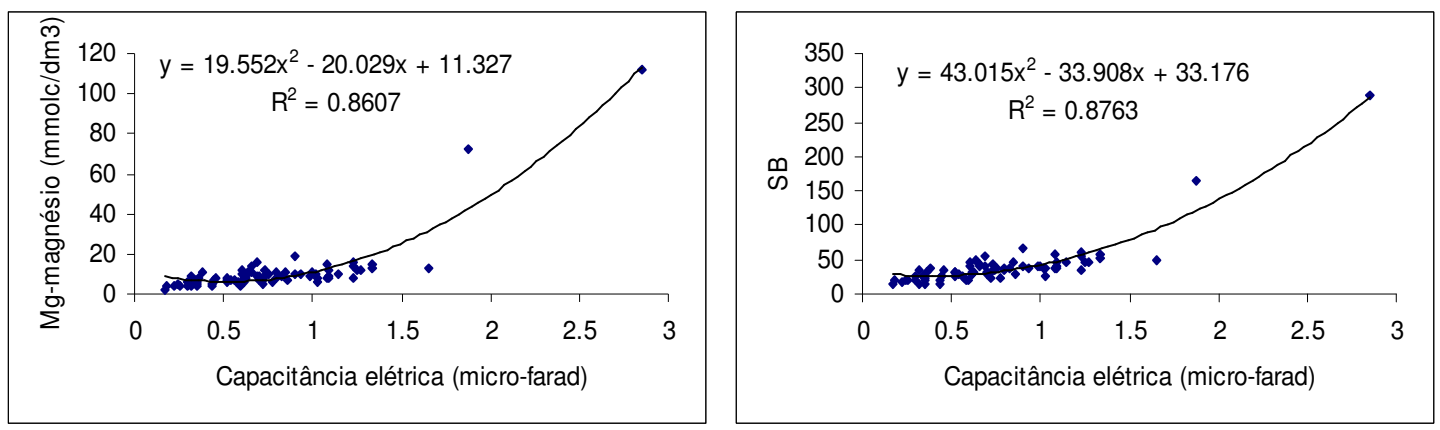

Figura 6 - Gráficos de dispersão e linha de tendência entre os valores de capacitância elétrica com magnésio (a) e soma de bases (b).

Na Figura 6, os coeficientes de determinação dos gráficos explicam com ótima segurança as variações do índice de magnésio e a soma de bases e a distribuição dos pontos nos gráficos se apresentam quase sem dispersão, indicando uma forte correlação entre as variáveis.
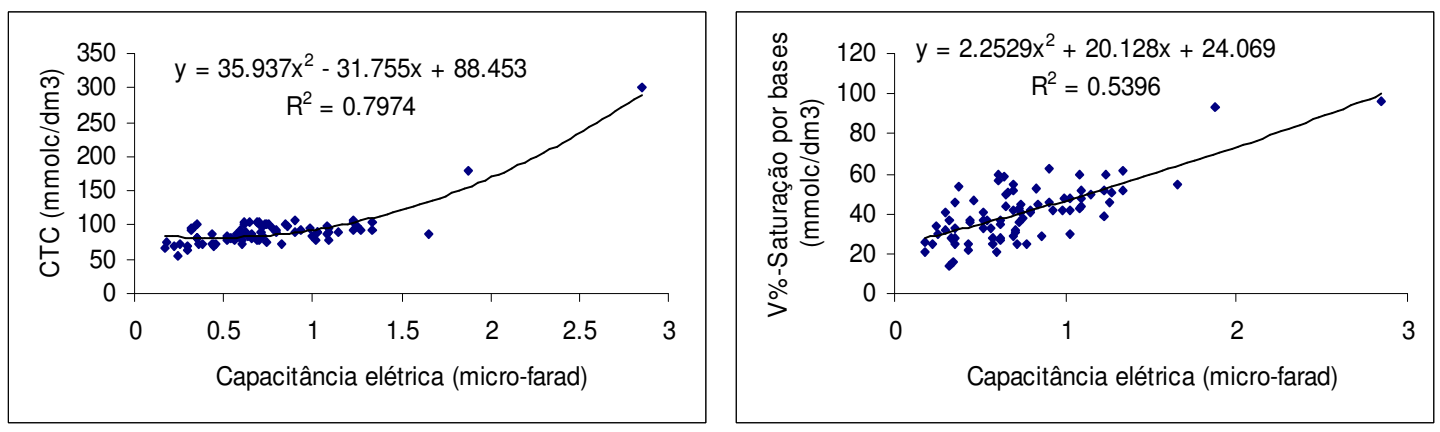

Figura 7 - Gráficos de dispersão e linha de tendência entre os valores de capacitância elétrica com CTC (a) e com V\% (b).

Na Figura 7 (a) pode-se visualizar que o coeficiente de determinação do gráfico explica com boa segurança as variações do índice de CTC e a distribuição dos pontos no gráfico se apresenta quase sem dispersão, indicando uma forte correlação entre as variáveis. Na Figura 7 (b) nota-se que o coeficiente de determinação do gráfico não explica com segurança as variações do índice de V\% e a distribuição dos pontos no gráfico se apresenta dispersa, indicando uma média correlação entre as variáveis. 

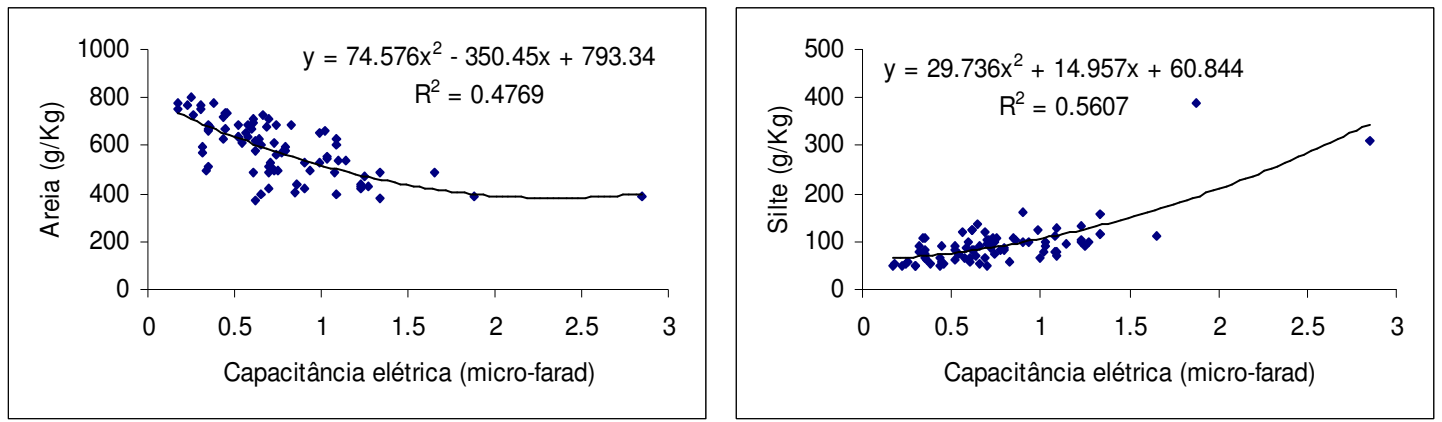

Figura 8 - Gráficos de dispersão e linha de tendência entre os valores de capacitância elétrica com os teores de areia (a) e com silte (b).

Pode-se observar na Figura 8 que os coeficientes de determinação de ambos os gráficos não explicam com segurança as variações do teor de areia e silte em função da capacitância elétrica e a distribuição dos pontos nos gráficos se apresentam dispersas, indicando uma média correlação entre as variáveis.

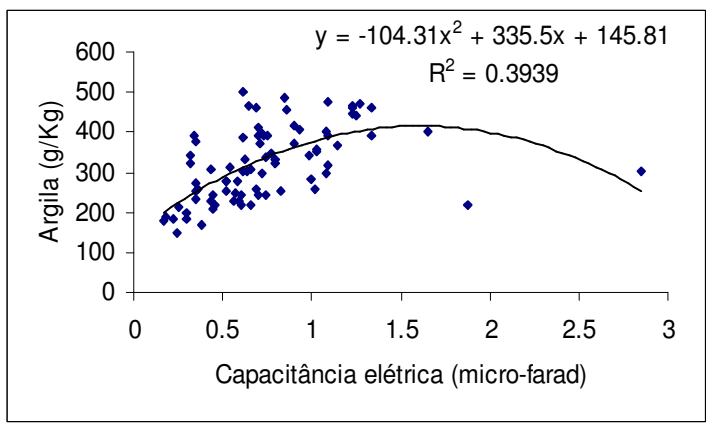

Figura 9 - Gráfico de dispersão e linha de tendência entre os valores de capacitância elétrica com os teores de argila.

Observa-se, na Figura 9, que o coeficiente de determinação do gráfico não explica com segurança as variações do teor de argila em função da capacitância elétrica e a distribuição dos pontos no gráfico se apresenta dispersa indicando uma média correlação entre as variáveis.

Pode-se notar nos gráficos de regressão que os melhores resultados de índices de correlação foram obtidos entre a capacitância elétrica com relação aos atributos do solo cálcio, magnésio, pH, CTC, soma de bases e o teor de silte, que apresentaram uma forte correlação, segundo classificação de (DORIA FILHO, 1999). 
Os melhores coeficientes de determinação foram obtidos entre capacitância elétrica e os atributos do solo cálcio, magnésio e soma de bases, que revelaram um ótimo percentual de ajustamento entre as variáveis, classificação de acordo com (VANNI, 1998).

\subsection{Análise de correlação entre os valores amostrados em campo e os valores estimados}

Utilizando os modelos matemáticos gerados pelas análises de regressão, foram calculados os valores estimados dos atributos do solo pelas medidas de capacitância elétrica do solo e comparados com os seus respectivos valores reais levantados no campo, para somente os maiores resultados de correlação entre as propriedades químicas do solo, e todas as propriedades físicas do solo observadas nas Figuras 2 a 9.

Tabela 1 - Resultados dos coeficientes de correlação (r) e determinação $\left(r^{2}\right)$ entre valor real e valor estimado pela análise de regressão com linha de tendência utilizando os valores de capacitância elétrica do solo.

\begin{tabular}{ccccccccc}
\hline & $\mathrm{pH}$ & $\mathrm{Ca}$ & $\mathrm{Mg}$ & $\mathrm{SB}$ & $\mathrm{CTC}$ & Areia & Silte & Argila \\
\hline $\mathrm{r}$ & 0,85 & 0,95 & 0,95 & 0,95 & 0,94 & 0,70 & 0,75 & 0,65 \\
$\mathrm{r}^{2}$ & 0,73 & 0,9 & 0,9 & 0,9 & 0,88 & 0,48 & 0,56 & 0,43 \\
\hline
\end{tabular}

De acordo com Vanni (1998), basicamente todo e qualquer coeficiente de correlação superior a $80 \%(0,80)$ revela que a relação é representativa dos pontos levantados. Sendo assim, destacam-se que nos resultados da Tabela 1, as correlações entre a capacitância elétrica e os atributos do solo pH, Ca, Mg, $\mathrm{SB}$ e CTC resultaram correlações acima de $80 \%$, revelando relações representativas entre os valores reais e os estimados. 


\section{CONCLUSÕES}

O sistema desenvolvido apresentou um baixo custo e a metodologia utilizada para a coleta de dados foi satisfatória, porém, evidenciou a necessidade de aprimorar a coleta de informação, buscando um aparelho que seja capaz de gravar as informações vindas do sensor, de modo que não seja necessária a planilha de anotações.

Para as condições em que esta pesquisa foi realizada, o sensor apresentou capacidade para medir as variações da capacitância elétricas do solo, correlacionando-se satisfatoriamente com os teores de argila e areia e fracamente com o teor de umidade. Isto demonstrou a possibilidade de uso do sensor para verificar a textura do solo em áreas não homogêneas, pois quanto maior o valor da capacitância elétrica do solo, a textura do mesmo tende a ser argilosa. Como o teor de umidade do solo é um fator que se associa com a textura do solo e como esta propriedade apresentou um índice baixo de correlação com a capacitância elétrica, não é recomendado o uso do sensor para determinar o teor de umidade do solo.

Os resultados verificados no presente estudo são promissores, pois demonstram que o sensor utilizado com o multímetro digital para medir capacitância elétrica do solo pode ser uma ferramenta útil no delineamento das unidades de gerenciamento e manejo localizado, no controle da fertilidade do solo para os seguintes atributos do solo: $\mathrm{pH}$, cálcio, magnésio, SB e CTC.

Novos estudos devem ser desenvolvidos para aprimorar os resultados encontrados na pesquisa.

\section{REFERÊNCIAS}

ADAMCHUK, V. I. et al. On -the-go soil sensors for precision agriculture. Computers and Electronics in Agriculture, Amsterdam, v. 44, p. 71-91, 2004.

DORIA FILHO, U. Introdução a bioestatística: para simples mortais. São Paulo: Negócio, 1999, 152 p.

EMBRAPA. Centro Nacional de Pesquisa de Solos. Manual de métodos de análise de solo. Rio de Janeiro, 1997. $212 \mathrm{p}$. 
GREEN, T. R.; SCHWANK, M.; FLUEHLER, H. Laboratory characterization of capacitance sensors for measuring soil water content. Fall Meeting of the Trans American Geophysical Union, San Francisco, CA, p. 5-9, Dec. 2005.

GREGO, C. R. et al. Variabilidade espacial da condutividade elétrica do solo e da produtividade da mamona (Ricinus communis L.) no sistema de plantio direto. In: CONGRESSO BRASILEIRO DE AGRICULTURA DE PRECISÃO, 2., 2006, São Pedro. Anais... Piracicaba: USP: ESALQ, 2006. 1 CD-ROM.

GUERRA, S. P. S. Desenvolvimento de um sistema informatizado de menor custo para aquisição e armazenamento de dados de sensores analógicos e receptor GPS. 2006. 118 f. Tese (Doutorado em Agronomia, Energia na Agricultura)-Faculdade de Ciências Agronômicas, Universidade Estadual Paulista, Botucatu, 2006.

PINCELLI, A. L. S. Desenvolvimento e ensaios de um sistema de mensuração de condutividade elétrica do solo. 2004. 96 f. Dissertação (Mestrado em Agronomia)-Escola Superior de Agricultura Luiz de Queiroz, Universidade de São Paulo, Piracicaba, 2004.

PLACIDO, M. E. B. Sistemas robotizados de inspeção interna de dutos. 2005. 130 f. Dissertação (Mestrado em Ciências)-Coordenação dos Programas de Pós-graduação de Engenharia, Universidade Federal de Rio de Janeiro, Rio de Janeiro, 2005.

RABELLO, L. M.; VAZ, C. M. P.; NETO, A. T. Sensor capacitivo para sondagem da umidade no perfil de solo. São Carlos : EMBRAPA, Instrumentação Agropecuária, 2005. 2 p. (Comunicado técnico, $71)$.

RAIJ, B. van. et al. Análise química para avaliação de fertilidade de solos tropicais. Campinas: Instituto Agronômico, 2001. 285 p.

RENDE, A.; BIAGE, M. Characterization of capacitive sensors for measurements of the moisture in irrigated soils. Journal of the Brazilian Society of Mechanical Sciences, Rio de Janeiro, v. 24, n. 3, Jul. 2002.

VANNI, S. M. Modelos de regressão : estatística aplica. São Paulo: Legmar Informática e Editora, 1998. $177 \mathrm{p}$. 